\title{
Coherent Light Harvesting through Strong Coupling to Confined Light
}

\author{
Gerrit Groenhof* ${ }^{* \dagger}$ a and J. Jussi Toppari \\ ${ }^{\dagger}$ Department of Chemistry and Nanoscience Center, P.O. Box 35, FIN-40014 University of Jyväskylä, Finland \\ ${ }^{\ddagger}$ Department of Physics and Nanoscience Center, P.O. Box 35, FIN-40014 University of Jyväskylä, Finland
}

\section{Supporting Information}

ABSTRACT: When photoactive molecules interact strongly with confined light modes, new hybrid light-matter states may form: the polaritons. These polaritons are coherent superpositions of excitations of the molecules and of the cavity photon. Recently, polaritons were shown to mediate energy transfer between chromophores at distances beyond the Förster limit. Here we explore the potential of strong coupling for light-harvesting applications by means of atomistic molecular dynamics simulations of mixtures of photoreactive and non-photo-reactive molecules strongly coupled to a single confined light mode. These molecules are spatially separated and present at different concentrations. Our simulations suggest that while the excitation is initially fully delocalized over all molecules and the confined

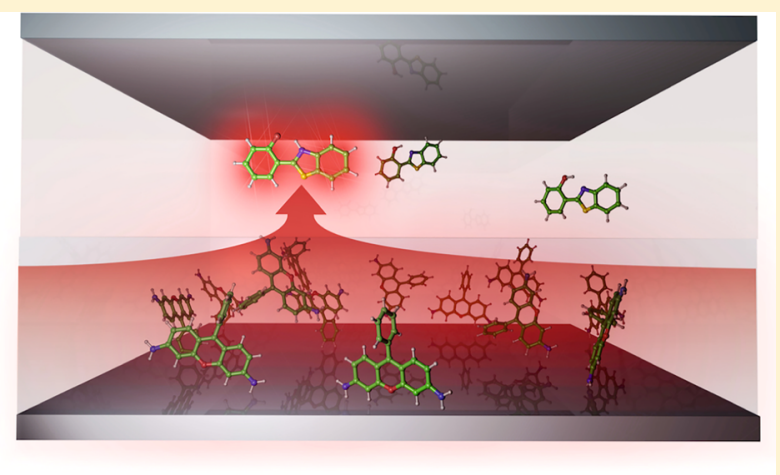
light mode, it very rapidly localizes onto one of the photoreactive molecules, which then undergoes the reaction.

$\mathrm{E}$ fficient excitation energy transfer, in which a photon is absorbed in one part of the system but utilized in another, is a key process in both natural and artificial light harvesting. ${ }^{1,2}$ Whereas the transfer usually occurs via the well-established Förster or Dexter mechanisms, ${ }^{3,4}$ recent experiments ${ }^{5-8}$ and theories $^{9-12}$ suggest that strong coupling of donor and acceptor molecules with a single confined light mode in an optical cavity can also mediate the transfer, even at distances beyond which the Förster mechanism can operate. ${ }^{7}$

Strong coupling between $N$ molecules and confined light leads to formation of $N+1$ hybrid light-matter states (socalled polaritons, ${ }^{13-18}$ Figure $\left.1 \mathrm{~b}\right)$ that are coherent superpositions of excitations in each of the molecules and of the confined light mode ${ }^{19,20}$

$$
\left.\psi_{K}=\sum_{i=1}^{N} \beta_{i}^{K}\left|g_{1} g_{2} \ldots e_{i} \ldots g_{N}\right\rangle|0\rangle+\alpha^{K}\left|g_{1} g_{2} \ldots g_{i} \ldots g_{N}\right| 1\right\rangle
$$

Here $\left|g_{i}\right\rangle$ and $\left|e_{i}\right\rangle$ are the ground and excited states of molecule $i$, while $|1\rangle$ or $|0\rangle$ indicates whether there is a photon in the cavity. The $\beta_{i}^{K}$ and $a^{K}$ are expansion coefficients, and the index $K$ labels the $N+1$ eigenstates of the system. When the excitation energy of the molecules $\left(\hbar \omega_{i}\right)$ is resonant with the frequency of the confined light mode $\left(\hbar \omega_{c}\right)$, the energy gap ( $\left.\hbar \Omega^{\mathrm{Rabi}}\right)$ between the lowest $(K=1$, i.e., the lower polariton) and highest $(K=N+1$, i.e., the upper polariton) hybrid state is proportional to the square root of the number of molecules $(N)$ that are strongly interacting with the confined light mode

$$
\hbar \Omega^{\mathrm{Rabi}}=2\left|\vec{\mu}_{\mathrm{m}}\right| \sqrt{N \hbar \omega_{\mathrm{c}} / \epsilon_{0} V_{\mathrm{c}}}
$$

where $\vec{\mu}_{\mathrm{m}}$ is the transition dipole moment of the molecules, $\epsilon_{0}$ is the vacuum permittivity, and $V_{\mathrm{c}}$ is the volume, in which the photon with (angular) frequency $\omega_{\mathrm{c}}$ is confined.

Because the lower $(K=1)$ and upper $(K=N+1)$ polaritons have much higher cross sections for light absorption than the other $N-1$ states, the absorption spectrum of the cavitymolecule system has two peaks instead of one, located below and above the absorption maxima of the cavity and the molecules (Figure 1c). In the lower and upper polaritons, the excitation is coherently shared between all molecules and the confined light mode, whereas in the other $N-1$ states, the excitation is more localized on subsets of the molecules.

Despite the delocalized character of the excitation into the lower polariton, recent simulations have shown that polaritons follow Kasha's rule and relax into the lowest energy state available to the cavity-molecule system. ${ }^{21,22}$ This observation suggests that if there are molecules that can undergo a photochemical reaction to energy levels below the lower polariton, then the coherently shared excitation can collapse and localize the excitation energy onto one of those molecules. Thus polaritonic light harvesting might be achieved if a large number of nonreactive chromophores are combined with a few photoreactive molecules within the volume of a confined light mode that is resonant with both types of molecules. In this situation, all molecules contribute to photon absorption,

Received: June 28, 2018

Accepted: August 7, 2018

Published: August 7, 2018 


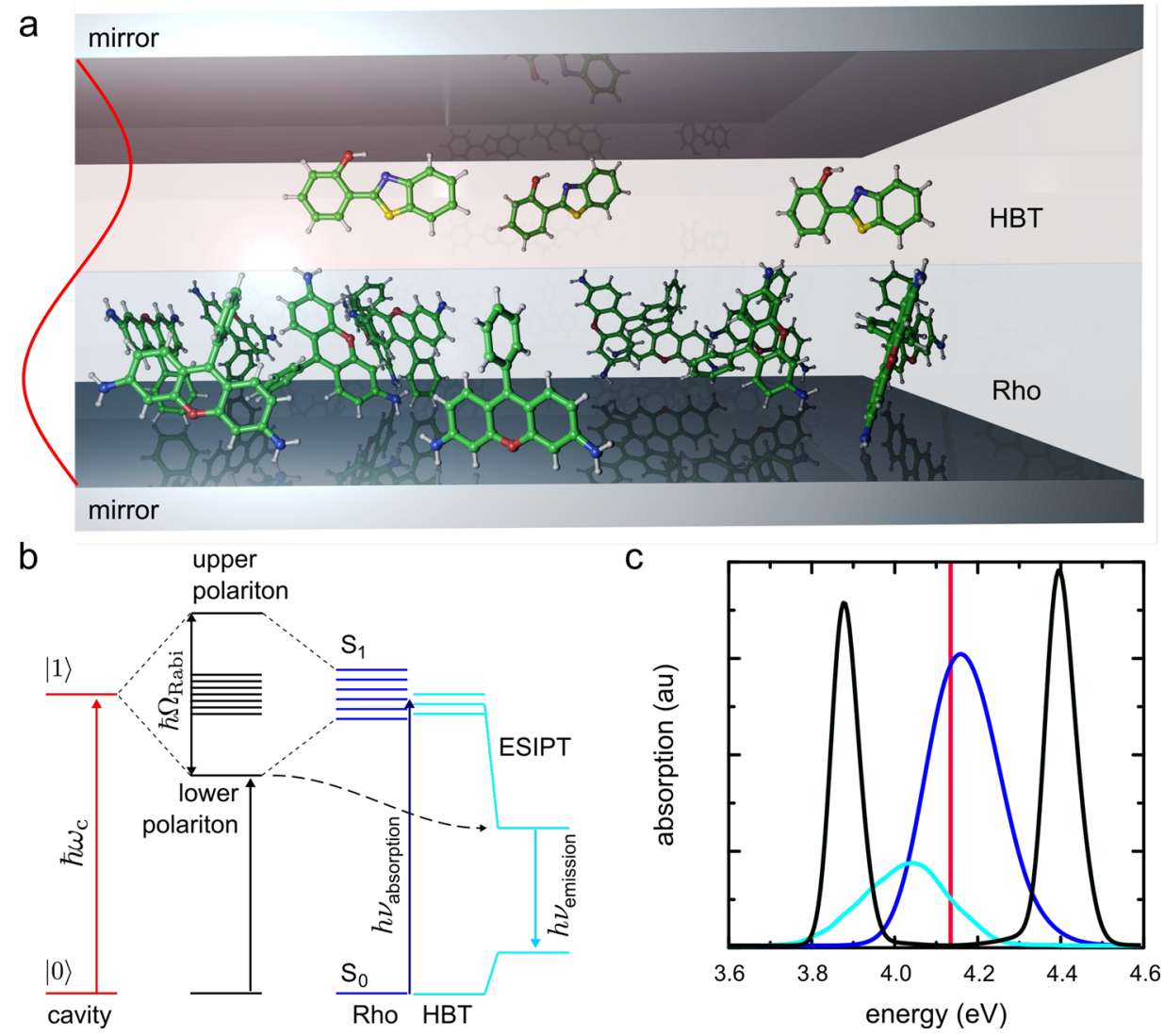

Figure 1. (a) Schematic depiction of the cavity-molecule system (not to scale). Inside the cavity there are two spatially separated layers of chromophores: rhodamine (Rho, blue) and hydroxyphenyl-benzothiazole (HBT, cyan). Because the second mode of the cavity $\left(\omega_{\mathcal{c}}\right.$, red) is resonant with the excitations of both chromophores, hybrid light-matter polaritons are formed (b). The dashed arrow in panel $b$ indicates that HBT could potentially undergo ultrafast intramolecular proton transfer (ESIPT) to a configuration with an energy below the lower polariton. Because of the red shift associated with this reaction, the HBT molecule is no longer in resonance with the cavity and can localize the excitation. (c) Polariton absorption spectrum (black) with 1000 Rho (blue) and 10 HBT molecules (cyan) strongly coupled to the confined light mode of the cavity (red).

whereas only one molecule eventually uses that energy for the reaction (Figure $1 \mathrm{~b}$ ).

Light can be efficiently confined between the mirrors of a Fabry-Pérot optical cavity ${ }^{23}$ or on the surface of metallic nanoparticles supporting a localized surface plasmon resonance (LSPR). ${ }^{24,25}$ Because in our simulations the details of the actual structure for confining light are irrelevant, ${ }^{21}$ our results will be valid for both types of light confinement. However, because of the high reflectivity of the mirrors, optical cavities are not very suited for light-harvesting applications. Therefore, practical realizations would more likely have to rely on open nanoparticle-based structures instead.

To test the idea, we constructed atomistic models with both reactive and nonreactive chromophores coupled to a single confined light mode that is resonant with their optical transitions. We assume that our cavity has an infinite quality (Q) factor. Thus a photon can be trapped between the mirrors forever and the cavity excitation never decays. To account for the effect of the light-matter coupling on the dynamics of the $N$ molecules, we followed Galego et al. ${ }^{26}$ to extend the traditional Jaynes-Cummings mode ${ }^{19,20}$ for molecules inside cavities. ${ }^{21}$ To obtain the $N+1$ polaritonic states (eq 1 ), the electronic ground and excited states of each molecule were calculated at the QM/MM level ${ }^{27}$ as well as their transition dipole moments. As explained in the Supporting Information (SI) and in ref 19, we use these energies and dipole moments to construct the light-matter Hamiltonian matrix that we diagonalize at every time step of the simulation to obtain the polaritonic potential energy surfaces and eigenstates. The dynamics of all molecules are propagated simultaneously on a single polaritonic potential energy surface, whereas transitions between the surfaces are modeled with a surface-hopping algorithm. $^{28}$

The very fast decay rate of cavity photons restricts the choice of photoreactive molecules to those with reaction rates within the polariton lifetime. Here we have selected 2-(2'hydroxyphenyl) benzothiazole (HBT), which undergoes ultrafast $(\sim 30 \mathrm{fs})$ excited-state intramolecular proton transfer (ESIPT) upon photoexcitation (Figure 2). ${ }^{29}$ This reaction is readily observed as a very large red shift in the emission from ultraviolet (UV, $340 \mathrm{~nm})$ to green $(525 \mathrm{~nm})$. In line with results from previous simulation studies ${ }^{30}$ and experiments, ${ }^{29}$

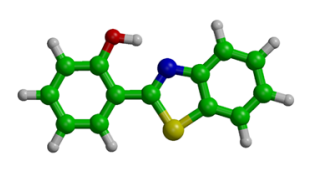

$\lambda_{\mathrm{abs}}=340 \mathrm{~nm}$

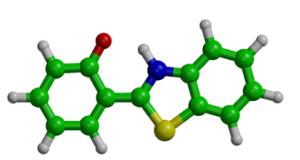

$\lambda_{\mathrm{em}}=525 \mathrm{~nm}$
Figure 2. Upon excitation with ultraviolet (UV) light at $340 \mathrm{~nm}, \mathrm{HBT}$ undergoes ultrafast intramolecular proton transfer, which causes the emission to red-shift to $525 \mathrm{~nm}$. Carbon atoms are green, oxygen is red, nitrogen is blue, sulfur is yellow and hydrogens are white. 
HBT undergoes proton transfer within tens of femtoseconds after photon absorption in QM/MM simulations (SI).

For the nonreactive molecules, we used the rhodamine model introduced in previous work. ${ }^{21}$ In this model, the rhodamine has an absorption maximum at $4.15 \mathrm{eV}$, which is close to the excitation maximum of HBT $(4.05 \mathrm{eV})$ in our simulations (Figure 1c). The simulated cavities contained up to 1000 rhodamines and $10 \mathrm{HBT}$ molecules in spatially separate layers, as in previous experiments. ${ }^{7}$ Both rhodamine and HBT layers were simultaneously coupled to the second confined light mode of the cavity with energy $\hbar \omega_{\mathrm{c}}=4.13 \mathrm{eV}$ (Figure 1a).

After ground-state equilibration, the combined moleculecavity system is resonantly excited into the optically active lower polariton at $3.9 \mathrm{eV}$ (Figure 1c). Initially, the excitation is fully delocalized over all molecules and the confined light mode of the cavity (Figure 3a). Because there is much more
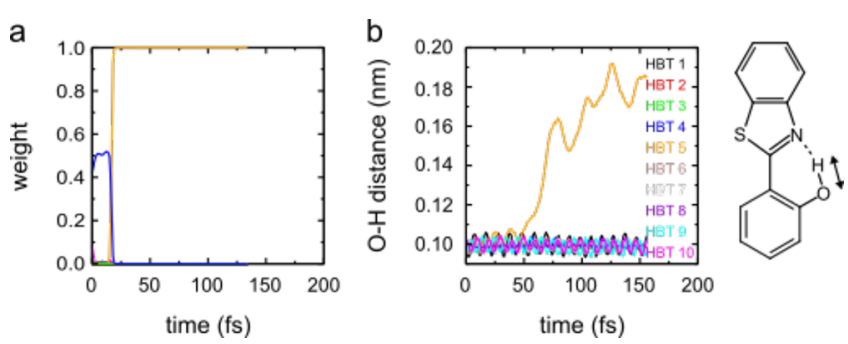

Figure 3. (a) Time evolution of the lowest energy polaritonic state during an MD simulation of $10 \mathrm{HBT}$ and 1000 Rho molecules in an optical cavity resonant with the molecular excitations. Plotted in panel $\mathrm{a}$ are the weights of the excitations on each molecule $\left(\left|\beta_{i}^{\mathrm{LP}}\right|^{2}\right.$ in all colors; $\left|\beta_{1005}^{\mathrm{LP}}\right|^{2}$, with the fifth HBT molecule excited, in orange) and cavity photon $\left(\left|\alpha^{\mathrm{LP}}\right|^{2}\right.$, blue, eq 1$)$. (b) Distance between the donor oxygen $(\mathrm{O})$ and proton $(\mathrm{H})$. About $30 \mathrm{fs}$ after localization of the excitation onto the fifth HBT molecule (orange), proton transfer to the nitrogen $(\mathrm{N})$ occurs, as evidenced by the increase in the $\mathrm{O}-\mathrm{H}$ distance. In the remaining nine $\mathrm{HBT}$ molecules, the $\mathrm{O}-\mathrm{H}$ distance fluctuates around the ground-state equilibrium bond length.

rhodamine than HBT and because rhodamine also has a larger transition dipole moment, the excitation mostly resides in the rhodamine layer (175:1). However, within $20 \mathrm{fs}$, the excitation localizes onto a single HBT molecule. After the collapse of the initially coherent excitation onto this HBT molecule, its hydroxyl proton transfers rapidly from the oxygen to the nitrogen atom, as shown in Figure $3 \mathrm{~b}$.

We repeated the simulations with various combinations of the molecules, keeping the $\mathrm{HBT} /$ rhodamine ratio identical. Also, in these simulations, the excitation is initially delocalized over all molecules but rapidly localizes onto one of the HBT molecules. These results, presented in the SI and summarized in Table 1, suggest that the variations have little effect on the efficiency of the excitation energy-transfer process under strong coupling. Because in most simulations the localization of the coherent excitation precedes proton transfer by several tens of femtoseconds, the photonic structures used for confining the light do not require long photon lifetimes or high $\mathrm{Q}$ factors. Therefore, if the energy levels, mode volumes and relaxation rates of the photonic structure are sufficient to reach the strong coupling limit for both molecules, then it is not relevant whether these are achieved with Fabry-Pérot cavities or with plasmonic surfaces, arrays or nanoparticles.

In summary, our atomistic simulations suggest that strong coupling can be utilized to achieve ultrafast and directional
Table 1. Number of Trajectories, Time of Excitation Localization, Time of Proton Transfer, and Rabi Splitting with Various Numbers of HBT and Rhodamine Molecules and Vacuum Field Strengths

\begin{tabular}{lccccc} 
HBT/Rho & trajectories & $\begin{array}{c}\text { vacuum } \\
\text { field }(\mathrm{au})^{a}\end{array}$ & $\begin{array}{c}\text { collapse } \\
(\mathrm{fs})\end{array}$ & $\begin{array}{c}\text { ESIPT } \\
(\mathrm{fs})^{b}\end{array}$ & $\begin{array}{c}\text { Rabi split } \\
(\mathrm{eV})^{c}\end{array}$ \\
\hline $10 / 1000$ & 1 & 0.00008 & $19^{d}$ & $65^{d}$ & 0.53 \\
$5 / 500$ & 2 & 0.00010 & $26 \pm 8$ & $83 \pm 25$ & 0.47 \\
$1 / 100$ & 10 & 0.00020 & $53 \pm 13$ & $145 \pm 22$ & 0.42
\end{tabular}

${ }^{a}$ Vacuum field is defined as $\sqrt{\hbar \omega_{c} / \epsilon_{0} V_{c}}$ (eq 2) in atomic units of energy and dipole moment and was chosen to yield a Rabi splitting of $\sim 0.5 \mathrm{eV}$ in all simulations. ${ }^{b}$ Time at which the $\mathrm{O}-\mathrm{H}$ distance exceeds $0.125 \mathrm{~nm}$. ${ }^{c}$ Rabi splitting at the start of the simulation. ${ }^{d}$ No error is estimated when there is only one trajectory.

transfer of excitation energy into a single photoreactive molecule. A layer of concentrated chromophores with large transition dipole moments inside a cavity or near a plasmonic nanostructure provides high oscillator strength, while a layer of low-concentration photoreactive molecules collects the energy necessary for their photochemical reactions. Although in this work, the acceptor of the excitation energy undergoes proton transfer, photovoltaic applications would aim for electron transfer instead. Via nanofabrication, such photo-oxidation reactions can be made to occur inside an optimal chemical environment, shielded from the other photoactive molecules, thereby preventing radiation damage or recombination.

\section{ASSOCIATED CONTENT}

\section{Supporting Information}

The Supporting Information is available free of charge on the ACS Publications website at DOI: 10.1021/acs.jpclett.8b02032.

Full methodological description of the cavity QM/MM simulations, QM/MM models and their validation, and tables and figures with data from all trajectories (PDF) QuickTime animation of excited-state intramolecular proton transfer in an isolated HBT molecule (MOV) QuickTime animation of excited-state intramolecular proton transfer in an HBT molecule that is inside a cavity with four other HBT and 500 Rho molecules (MOV)

\section{AUTHOR INFORMATION}

\section{Corresponding Author}

*E-mail: gerrit.x.groenhof@jyu.fi.

ORCID

Gerrit Groenhof: 0000-0001-8148-5334

Notes

The authors declare no competing financial interest.

\section{ACKNOWLEDGMENTS}

We thank Professor Thomas Ebbesen for raising our interest in this subject. We also thank CSC-IT Center for Science for generous computational resources as well as PRACE for awarding us access to resource Curie based in France at GENCI. This work was supported by the Academy of Finland (grants 290677 to G.G. and 289947 to J.J.T.). 


\section{REFERENCES}

(1) Croce, R.; van Amerongen, H. Natural strategies for photosynthetic light harvesting. Nat. Chem. Biol. 2014, 10, 492-501.

(2) Harriman, A. Artificial light-harvesting arrays for solar energy conversion. Chem. Commun. 2015, 51, 11745-11756.

(3) Förster, T. Zwischenmolekulare Energiewanderung und Fluoreszenz. Ann. Phys. 1948, 437, 55-75.

(4) Dexter, D. L. A theory of sensitized luminescence in solids. J. Chem. Phys. 1953, 21, 836-850.

(5) Coles, D. M.; Somaschi, N.; Michetti, P.; Clark, C.; Lagoudakis, P. G.; Savvidis, P. G.; Lidzey, D. G. Polariton-mediated energy transfer between organic dyes in a strongly coupled optical microcavity. Nat. Mater. 2014, 13, 712-719.

(6) Zhong, X.; Chervy, T.; Wang, S.; George, J.; Thomas, A.; Hutchison, J. A.; Devaux, E.; Genet, C.; Ebbesen, T. W. Non-radiative energy transfer mediated by hybrid light-matter states. Angew. Chem., Int. Ed. 2016, 55, 6202-6206.

(7) Zhong, X.; Chervy, T.; Zhang, L.; Thomas, A.; George, J.; Genet, C.; Hutchison, J. A.; Ebbesen, T. W. Energy transfer between spatially separated entangled molecules. Angew. Chem., Int. Ed. 2017, 56, 9034-9038.

(8) Akulov, K.; Bochman, D.; Golombek, A.; Schwartz, T. Longdistance resonant energy transfer mediated by photonic modes. $J$. Phys. Chem. C 2018, 122, 15853.

(9) Feist, J.; Garcia-Vidal, F. J. Extraordinary Exciton Conductance Induced by Strong Coupling. Phys. Rev. Lett. 2015, 114, 196402.

(10) Schachenmayer, J.; Genes, C.; Tignone, E.; Pupillo, G. Cavityenhanced transport of excitons. Phys. Rev. Lett. 2015, 114, 196403.

(11) Reitz, M.; Mineo, F.; Genes, C. Energy transfer and correlations in cavity-embedded donor-acceptor configurations. Sci. Rep. 2018, 8, 9050.

(12) Du, M.; Martinez, L. A.; Ribeiro, R. F.; Hu, Z.; Menon, V.; Yuen-Zhou, J. Theory for polariton-assisted remote energy transfer. 2018, arXiv:1711.11576. arXiv.org e-Print archive. https://arxiv.org/ abs/1711.11576.

(13) Lidzey, D. G.; Bradley, D. D. C.; Skolnick, M. S.; Virgili, T.; Walker, S.; Whittaker, D. M. Strong exciton-photon coupling in an organic semiconductor microcavity. Nature 1998, 395, 53-55.

(14) Hutchison, J. A.; Schwartz, T.; Genet, C.; Devaux, E.; Ebbesen, T. W. Modifying chemical energy landscapes by coupling to vacuum fields. Angew. Chem., Int. Ed. 2012, 51, 1592-1596.

(15) Ebbesen, T. W. Hybrid light-matter states in a molecular and material science perspective. Acc. Chem. Res. 2016, 49, 2403-2412.

(16) George, J.; Wang, S.; Chervy, T.; Canaguier-Durand, A.; Schaeffer, G.; Lehn, J.-M.; Hutchison, J. A.; Genet, C.; Ebbesen, T. W. Ultra-strong coupling of molecular materials: spectroscopy and dynamics. Faraday Discuss. 2015, 178, 281-294.

(17) Chikkaraddy, R.; De Nijs, B.; Benz, F.; Barrow, S. J.; Scherman, O. A.; Rosta, E.; Demetriadou, A.; Fox, P.; Hess, O.; Baumberg, J. J. Single-molecule strong coupling at room temperature in plasmonic nanocavities. Nature 2016, 535, 127-130.

(18) Dovzhenko, D. S.; Ryabchuk, S. V.; Rakovich, Y. P.; Nabiev, I. R. Light-matter interaction in the strong coupling regime: configurations, conditions, applications. Nanoscale 2018, 10, 35893605.

(19) Jaynes, E. T.; Cummings, F. W. Comparison of quantum and semiclassical radiation theories with application to the beam maser. Proc. IEEE 1963, 51, 89-109.

(20) Tavis, M.; Cummings, F. W. Exact solution for an N-moleculeradiation-field Hamiltonian. Phys. Rev. 1969, 188, 692-695.

(21) Luk, H. L.; Feist, J.; Toppari, J. J.; Groenhof, G. Multi-scale molecular dynamics simulations of polaritonic chemistry. J. Chem. Theory Comput. 2017, 13, 4324-4335.

(22) Baieva, S.; Hakamaa, O.; Groenhof, G.; Heikkilä, T.; Toppari, J. J. Dynamics of Strongly Coupled Modes between Surface Plasmon Polaritons and Photoactive Molecules: The Effect of the Stokes Shift. ACS Photonics 2017, 4, 28-37.

(23) Vahala, K. J. Optical microcavities. Nature 2003, 424, 839-846.
(24) Törmä, P.; Barnes, W. L. Strong coupling between surface plasmon polaritons and emitters: a review. Rep. Prog. Phys. 2015, 78, 013901.

(25) Zengin, G.; Wersäll, M.; Nilsson, S.; Antosiewicz, T. J.; Käll, M.; Shegai, T. Realizing strong light-matter interactions between single-nanoparticle plasmons and molecular excitations at ambient conditions. Phys. Rev. Lett. 2015, 114, 157401.

(26) Galego, J.; Garcia-Vidal, F. J.; Feist, J. Cavity-induced modifications of molecular structure in the strong coupling limit. Phys. Rev. X 2015, 5, 041022.

(27) Warshel, A.; Levitt, M. Theoretical studies of enzymic reactions: dielectric, electrostatic and steric stabilization of the carbonium ion in the reaction of lysozyme. J. Mol. Biol. 1976, 103, 227-249.

(28) Tully, J. C.; Preston, R. K. Trajectory surface hopping approach to nonadiabatic molecular collisions: the reaction of $\mathrm{H}^{+}$with $\mathrm{D}_{2} . J$. Chem. Phys. 1971, 55, 562-572.

(29) Lochbrunner, S.; Wurzer, A. J.; Riedle, E. Microscopic mechanism of ultrafast excited-state intramolecular proton transfer: a 30-fs study of 2-(2'-hydroxyphenyl) bezothiazole. J. Phys. Chem. A 2003, 107, 10580-10590.

(30) Pijeau, S.; Foster, D.; Hohenstein, E. G. Excited-state dynamics of 2-(2'-hydroxyphenyl) benzathiazole: ultrafast proton transfer and internal conversion. J. Phys. Chem. A 2017, 121, 4595-4605. 